\title{
1-S07-4 Symposium
}

\section{The actin cytoskeleton in cardiomyocytes: implications for cardiac function}

\section{$\underline{\text { Ryu Takeya }}^{1}$}

${ }^{I}$ Dept. Pharmacol., Fac. Med., Univ. of Miyazaki

Contraction of cardiac muscle is caused by periodic sliding of an array of thin actin filaments into a lattice of thick myosin filaments in the sarcomere, the contractile unit of striated muscles. In actively contracting cardiomyocytes, thin filaments exhibit continuous exchange of actin subunits at their ends, although underlying mechanisms are not well understood. Fhod3, a cardiac member of the formin family proteins, is a likely candidate for a key regulator of actin dynamics in cardiomyocytes. We have shown that Fhod3 is required for cardiac development and the maintenance of the normal contractile function of the heart. Here we show a direct molecular link between Fhod3 and cMyBP-C, a thick myosin filament-associated protein that modulates myocardial contraction via cross-bridge arrangement. The direct interaction between Fhod3 and cMyBP-C appears to serve to control the Fhod3-mediated actin turnover in a manner that depends on cross-bridge arrangement. Indeed, overexpression of Fhod 3 in the absence of cMyBP-C adversely affected cardiac function with a defect of sarcomere integrity. We will discuss the underlying mechanism and also discuss the possibility that targeted disruption of this cross-talk machinery leads to an artificial modulation of cardiac contractility. 